\title{
Siacci's resolution of the acceleration vector for a space curve
}

\author{
James Casey
}

Received: 17 January 2009 / Accepted: 16 March 2010 / Published online: 9 June 2010

(C) The Author(s) 2010. This article is published with open access at Springerlink.com

\begin{abstract}
For motion of a material point along a space curve, a kinematical decomposition, discovered by Siacci, expresses the acceleration vector as the sum of two special oblique components in the osculating plane to the curve. A new proof of Siacci's theorem is presented.
\end{abstract}

Keywords Siacci - Space curve $\cdot$ Kinematics .

Classical mechanics $\cdot$ Central forces

\section{Introduction}

For planar motions of a particle, a resolution of the acceleration vector, due to Siacci [1], is well known and is particularly useful for problems in which angular momentum is constant [2-6]. This resolution comprises a radial component and a tangential component, which, in general, are not orthogonal to each other. ${ }^{1}$

\footnotetext{
${ }^{1}$ Whittaker [2, p. 21] gives a geometrical proof of Siacci's theorem in the plane. Grossman [3] has a more modern proof (but the accompanying diagram is misdrawn). Francesco Siacci (18391907 ) held professorships in ballistics at the military academy in Genoa, and in mechanics at the Universities of Turin and Naples [7]. He played an active role in the movement for unification of Italy, and in his later years was elected a Senator for life. A street in Rome is named in his honor.
}

J. Casey $(\bowtie)$

Department of Mechanical Engineering, University

of California, Berkeley, CA 94720, USA

e-mail: jcasey@me.berkeley.edu
For spatial motions of a particle, Siacci succeeded in showing that a similar resolution can be obtained [8]. In this case, the two components are in the instantaneous osculating plane to the path of the particle. One is tangent to the path, while the other is directed towards the point where the perpendicular from a fixed origin meets the osculating plane. Although Siacci's formulas are quite remarkable, his statement of the theorem is not altogether precise and his proof is cumbersome. $^{2}$

The purpose of this note is to present a proof of Siacci's theorem in space that is based on the SerretFrenet formulae. Siacci's theorem in the plane is included as a corollary.

\section{Preliminaries}

Consider a particle $P$ of mass $m(>0)$ moving in a 3-dimensional Euclidean space $\mathbb{E}^{3}$ under the influence of arbitrary forces. Choose an arbitrary fixed origin $O$ in $\mathbb{E}^{3}$, and let $\mathbf{x}$ be the position vector of $P$ at time $t$. Let $\mathcal{C}$ be the oriented curve traced out by $P$, and let $s$ be the arc length of $\mathcal{C}$ corresponding to time $t$.

Let $\left\{\mathbf{e}_{t}, \mathbf{e}_{n}, \mathbf{e}_{b}\right\}$ be a right-handed orthonormal basis consisting of the unit tangent vector

$\mathbf{e}_{t}=\mathbf{x}^{\prime}=\frac{d \mathbf{x}}{d s}$,

\footnotetext{
${ }^{2}$ Whittaker [2, p. 24, Problem 12] repeats Siacci's statement of the theorem.
} 
the unit normal vector $\mathbf{e}_{n}$, and the unit binormal vector $\mathbf{e}_{b}$. Recall the Serret-Frenet formulae

$\mathbf{e}_{t}^{\prime}=\kappa \mathbf{e}_{n}, \quad \mathbf{e}_{n}^{\prime}=-\kappa \mathbf{e}_{t}+\tau \mathbf{e}_{b}, \quad \mathbf{e}_{b}^{\prime}=-\tau \mathbf{e}_{n}$,

where $\kappa$ and $\tau$ are the curvature and torsion functions. For $\kappa \neq 0, \rho=1 / \kappa$ is the radius of curvature of $\mathcal{C}$.

The velocity $\mathbf{v}$ and acceleration $\mathbf{a}$ of $P$ at time $t$ are given by

$\mathbf{v}=\dot{\mathbf{x}}=v \mathbf{e}_{t}, \quad v=\dot{s}, \quad \mathbf{a}=\dot{\mathbf{v}}=\dot{v} \mathbf{e}_{t}+\kappa v^{2} \mathbf{e}_{n}$,

where the superposed dot denotes $d / d t$. Along $\mathcal{C}, \mathbf{v}$ may be expressed as a function of $s$, and the acceleration may then be written as

$\mathbf{a}=v \frac{d v}{d s} \mathbf{e}_{t}+\kappa v^{2} \mathbf{e}_{n}$,

which always lies in the osculating plane $\Pi$ to $\mathcal{C}$ at $P$. The angular velocity vector of the Serret-Frenet basis is

$\omega=\dot{s}\left(\tau \mathbf{e}_{t}+\kappa \mathbf{e}_{b}\right)$

and lies in the rectifying plane to $\mathcal{C}$. Clearly,

$\dot{\mathbf{e}}_{t}=\omega \times \mathbf{e}_{t}, \quad \dot{\mathbf{e}}_{n}=\omega \times \mathbf{e}_{n}, \quad \dot{\mathbf{e}}_{b}=\omega \times \mathbf{e}_{b}$.

The angular momentum of $P$ about $O$ is

$\mathbf{H}^{O}=\mathbf{x} \times m \mathbf{v}=\mathbf{x} \times m v \mathbf{e}_{t}$

and lies in the plane perpendicular to $\mathbf{e}_{t}$ (i.e., the normal plane to $\mathcal{C}$ ).

\section{Siacci's theorem}

Let the position vector of $P$ be resolved on the SerretFrenet basis:

$\mathbf{x}=q \mathbf{e}_{t}-p \mathbf{e}_{n}+b \mathbf{e}_{b}$,

where

$q=\mathbf{x} \cdot \mathbf{e}_{t}, \quad-p=\mathbf{x} \cdot \mathbf{e}_{n}, \quad b=\mathbf{x} \cdot \mathbf{e}_{b}$.

Also, define a vector $\mathbf{r}$ in the osculating plane $\Pi$ by

$\mathbf{r}=-p \mathbf{e}_{n}+q \mathbf{e}_{t}$.

Note that

$r^{2}=\mathbf{r} \cdot \mathbf{r}=p^{2}+q^{2}$.
The foregoing resolution of $\mathbf{x}$ is indicated in Fig. 1 . The vector $b \mathbf{e}_{b}$ is the position vector of the perpendicular $O B$ from $O$ to the osculating plane, and $\mathbf{r}$ is the position vector of $P$ relative to $B . B Z$ is the perpendicular from $B$ to the tangent line $S P T$, so that the position vector of $Z$ relative to $B$ is $-p \mathbf{e}_{n}$. The position vector of $P$ relative to $Z$ is $q \mathbf{e}_{t}$.

Substituting (8) in (1) and invoking (2) $1,2,3$, we obtain

$$
\begin{aligned}
\mathbf{e}_{t}= & q^{\prime} \mathbf{e}_{t}+q \kappa \mathbf{e}_{n}-p^{\prime} \mathbf{e}_{n}-p\left(-\kappa \mathbf{e}_{t}+\tau \mathbf{e}_{b}\right)+b^{\prime} \mathbf{e}_{b} \\
& +b\left(-\tau \mathbf{e}_{n}\right),
\end{aligned}
$$

which yields

$q^{\prime}=1-\kappa p, \quad p^{\prime}=\kappa q-\tau b, \quad b^{\prime}=\tau p$.

It follows from (11) and (13) $1,2,3$ that

$r r^{\prime}=q-\tau p b=q-b b^{\prime}$.

With the aid of (8), the angular momentum vector in (7) takes the form

$\mathbf{H}^{O}=m\left(b \mathbf{e}_{n}+p \mathbf{e}_{b}\right) v$.

Let

$h=p v, \quad w=b v$.

The two components, $h$ and $w$, of angular momentum per unit mass may be interpreted as twice the rates at which the orthogonal projections of $\mathbf{x}$ sweep out area on the osculating plane $\Pi$ and on the rectifying plane, respectively. ${ }^{3}$

Siacci's idea is to resolve the acceleration vector a in (4) along the tangential direction $S P T$ and the radial direction $B P$ in the osculating plane. To do this, we need to be able to express $\mathbf{e}_{n}$ in terms of $\mathbf{r}$ and $\mathbf{e}_{t}$, which in view of (10), is possible if and only if $p \neq$ 0 . We may ensure that $p$ never vanishes by making the physical assumption that the binormal component of angular momentum is nonzero. Then, $h=p v \neq 0$. Hence, in view of (11), $r \neq 0$ and we may define a unit vector $\mathbf{e}_{r}$ by

$\mathbf{e}_{r}=\frac{1}{r} \mathbf{r}$

\footnotetext{
${ }^{3}$ See [9] for a discussion of the areal velocity vector in space.
} 


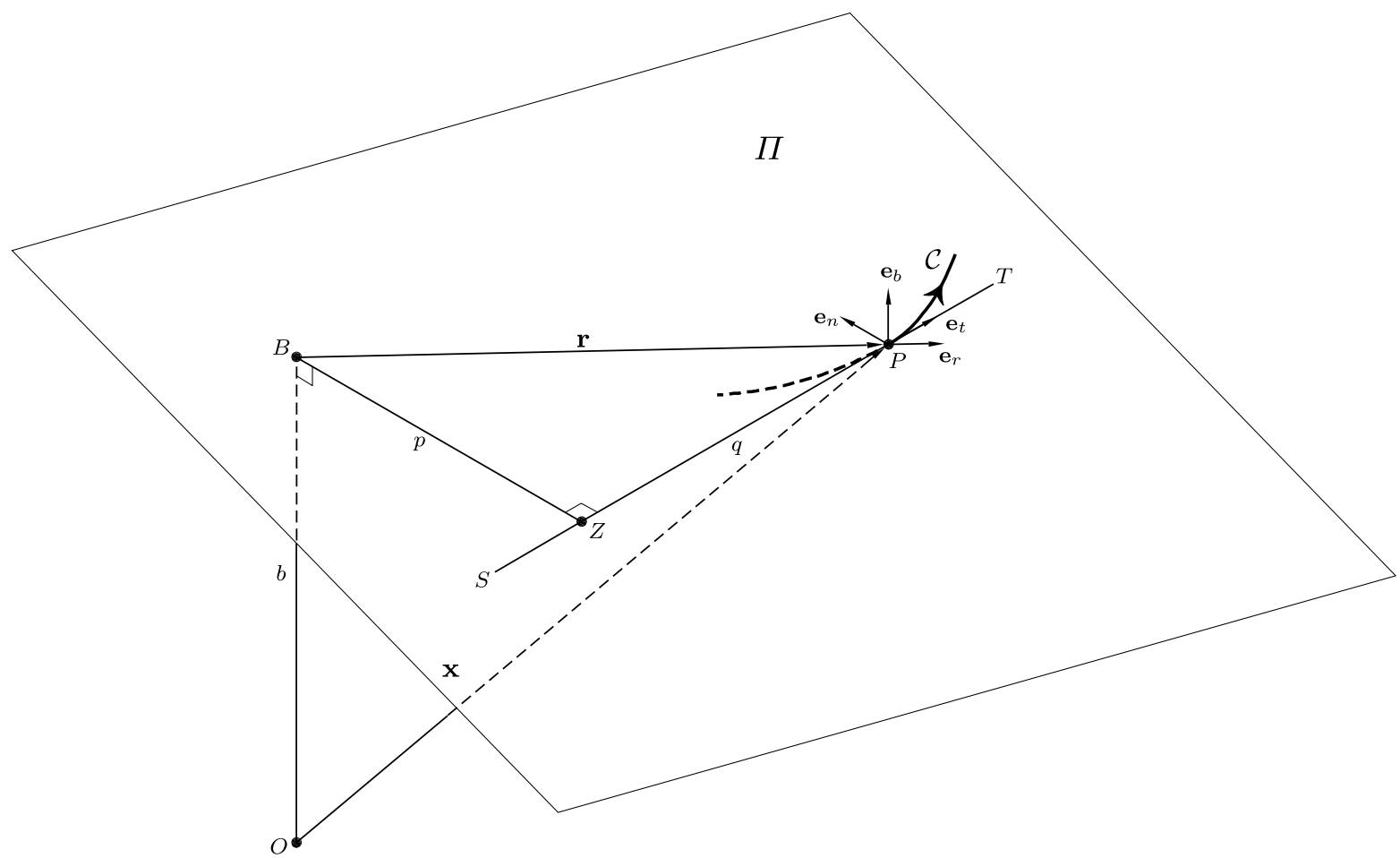

Fig. 1 A particle $P$ travels along a space curve $\mathcal{C}$. $S P T$ is the tangent line to $\mathcal{C}$ at $P$ and $\Pi$ is the osculating plane at $P$. $O B$ is the perpendicular, from an arbitrary fixed origin $O$ in space,

Equation (10) then yields the desired resolution

$\mathbf{e}_{n}=\frac{1}{p}\left(-r \mathbf{e}_{r}+q \mathbf{e}_{t}\right)$.

Substituting (18) in (4), we obtain the fundamental form of Siacci's resolution of the acceleration vector:

$\mathbf{a}=-\frac{\kappa v^{2} r}{p} \mathbf{e}_{r}+\left(v \frac{d v}{d s}+\frac{\kappa v^{2} q}{p}\right) \mathbf{e}_{t}=S_{r} \mathbf{e}_{r}+S_{t} \mathbf{e}_{t}$

In general, the unit vectors $\mathbf{e}_{r}$ and $\mathbf{e}_{t}$ are not orthogonal to each other and the two components of $\mathbf{a}$ in (19) are not equal to the orthogonal projections of $\mathbf{a}$ onto $\mathbf{e}_{r}$ and $\mathbf{e}_{t}$. We call $S_{r}$ and $S_{t}$, respectively, the radial and tangential Siacci components of the acceleration.

Invoking (16) 1 with $p \neq 0$, we may express the radial Siacci component as

$S_{r}=-\frac{\kappa r h^{2}}{p^{3}}$ to $\Pi$. $B Z$ is perpendicular to $S P T$, and $\mathbf{r}$ is the position vector of $P$ relative to $B$

The tangential Siacci component may be put into a number of different forms. First note that $q$ satisfies the equations

$\kappa q=p^{\prime}+\tau b, \quad q=r r^{\prime}+\tau p b=r r^{\prime}+b b^{\prime}$,

which follow from (13) 2 and (14). With the aid of $(21)_{1},(16)_{1}$ and $(13)_{3}$, we find that

$$
\begin{aligned}
S_{t} & =\frac{1}{2}\left(v^{2}\right)^{\prime}+\frac{\kappa v^{2} q}{p} \\
& =\frac{1}{2}\left(v^{2}\right)^{\prime}+\frac{v^{2} p^{\prime}}{p}+\frac{\tau v^{2} b}{p} \\
& =\frac{\left(h^{2}\right)^{\prime}}{2 p^{2}}+\frac{\tau b h^{2}}{p^{3}} \\
& =\frac{1}{2 p^{2}}\left\{\left(h^{2}\right)^{\prime}+\frac{h^{2}}{p^{2}}\left(b^{2}\right)^{\prime}\right\} .
\end{aligned}
$$

Likewise, employing $(21)_{2}$, we obtain

$$
S_{t}=\frac{1}{2}\left(v^{2}\right)^{\prime}+\kappa v^{2}\left\{\frac{\left(r^{2}\right)^{\prime}}{2 p}+\tau b\right\} \text {. }
$$


If instead, we use the second expression for $q$ in $(21)_{2}$, we may deduce that

$$
\begin{aligned}
S_{t} & =\frac{1}{2}\left(v^{2}\right)^{\prime}+\frac{\kappa v^{2}}{2 p}\left(r^{2}+b^{2}\right)^{\prime} \\
& =\frac{1}{2}\left(v^{2}\right)^{\prime}+\frac{\kappa v^{2}}{2 p}(\mathbf{x} \cdot \mathbf{x})^{\prime},
\end{aligned}
$$

where (10) and (8) have also been utilized.

Summarizing the above derivation, we state

Siacci's Theorem Let a particle $P$ of mass $m(>0)$ travel along a curve $\mathcal{C}$ in space, and suppose that the binormal component of its angular momentum vector never vanishes. Then, the acceleration vector of $P$ may be expressed as the sum of two oblique components, the Siacci components, as in (19). One Siacci component lies along the tangent to $\mathcal{C}$, while the other one is directed from $P$ towards the point where the perpendicular from an arbitrary fixed origin meets the osculating plane. The radial component may also be written as in (20), and alternative expressions for the tangential component are given by (22), (23), and (24).

Corollary 1 Suppose that the motion of $P$ is confined to a fixed plane, not necessarily containing the origin $O$, and that the component of angular momentum that is perpendicular to the plane never vanishes. Then, the acceleration of $P$ can be expressed in the form (19), where $\mathbf{e}_{r}$ is the radial basis vector of a polar coordinate system in the plane. The tangential Siacci component reduces to

$$
S_{t}=\frac{\left(h^{2}\right)^{\prime}}{2 p^{2}},
$$

or equivalently to

$$
S_{t}=\frac{1}{2}\left\{\left(v^{2}\right)^{\prime}+\frac{\kappa v^{2}}{p}\left(r^{2}\right)^{\prime}\right\} .
$$

Proof In the planar case, $\tau=0, \mathbf{e}_{b}$ is constant and orthogonal to the plane, and $b$ is constant. Also, the component $m p v \mathbf{e}_{b}$ of angular momentum in (15) is orthogonal to the plane, and, by assumption, never vanishes. By virtue of (8), (10) and (11) the position vector of $P$ is

$\mathbf{x}=r \mathbf{e}_{r}+b \mathbf{e}_{b}$
The expression (19) now holds in the plane of the motion. Equations (25) and (26) follow immediately from (22) and (23).

It may be noted that in the planar case, (14) and $(13)_{2}$ yield

$r r^{\prime}=q, \quad p^{\prime}=\kappa r r^{\prime}$.

Corollary 2 Suppose that the fixed plane in Corollary 1 passes through $O$, and that the angular momentum of $P$ never vanishes. Then, Siacci's resolution (19) holds, with $S_{t}$ being given by (25) and (26).

Proof This is a special case of Corollary 1 with $b=0$.

\section{Illustrative examples}

Example 1: Motion along a helix

Let the curve $\mathcal{C}$ be a circular helix, along which the particle $P$ is traveling. The helix lies on a cylinder of radius $A$.

Using a cylindrical coordinate system $(R, \theta, z)$, let the position vector of $P$ be given by

$\mathbf{x}=A \mathbf{e}_{R}+z \mathbf{k}, \quad z=B \theta$,

where $\mathbf{e}_{R}=\cos \theta \mathbf{i}+\sin \theta \mathbf{j}, \mathbf{e}_{\theta}=-\sin \theta \mathbf{i}+\cos \theta \mathbf{j}$, $\{\mathbf{i}, \mathbf{j}, \mathbf{k}\}$ is a fixed right-handed orthonormal basis, and $A(>0)$ and $B(\neq 0)$ are constants. Let $\omega=\dot{\theta}$. The velocity and acceleration vectors of $P$ are

$\mathbf{v}=\omega\left(A \mathbf{e}_{\theta}+B \mathbf{k}\right)$,

$\mathbf{a}=-A \omega^{2} \mathbf{e}_{R}+\dot{\omega}\left(A \mathbf{e}_{\theta}+B \mathbf{k}\right)$.

Let $C=\sqrt{A^{2}+B^{2}}$. The speed of $P$ and its time derivative are

$v=\dot{s}=C \omega, \quad v \frac{d v}{d s}=C \dot{\omega}$.

The arc length increases linearly with $\theta$.

The Serret-Frenet basis for the helix is

$\mathbf{e}_{t}=\cos \alpha \mathbf{e}_{\theta}+\sin \alpha \mathbf{k}, \quad \mathbf{e}_{n}=-\mathbf{e}_{R}$,

$\mathbf{e}_{b}=-\sin \alpha \mathbf{e}_{\theta}+\cos \alpha \mathbf{k}$, 
where the helix angle $\alpha$ is given by $\tan \alpha=B / A$. The curvature and torsion are constant:

$\kappa=\frac{A}{C^{2}}=\frac{\cos ^{2} \alpha}{A}, \quad \tau=\frac{B}{C^{2}}=\kappa \tan \alpha$.

The angular velocity of the Serret-Frenet basis is

$\omega=\omega \mathbf{k}$.

The components of $\mathbf{x}$ on the Serret-Frenet basis may be calculated from $(9)_{1,2,3},(29)_{1}$, and $(32)_{1,2,3}$ :

$q=z \sin \alpha, \quad p=A, \quad b=z \cos \alpha$.

The two components of the angular momentum per unit mass, given by $(16)_{1,2}$, are

$h=A C \omega, \quad w=A z \omega$.

Applying Siacci's theorem in space, we find that

$S_{r}=-r \omega^{2}=-\omega^{2} \sqrt{A^{2}+z^{2} \sin ^{2} \alpha}$,
$S_{t}=C \dot{\omega}+\omega^{2} z \sin \alpha$.

\section{Example 2: Central forces}

Suppose that the hypotheses of Corollary 2 are satisfied. Suppose also that the angular momentum of $P$ is a nonzero constant and that the radial component $S_{r}$ of acceleration is a function of $r$ :

$S_{r}=-f(r)$.

The tangential Siacci component of acceleration in (25) now vanishes. From (20) and (38), it follows that

$\frac{\kappa r h^{2}}{p^{3}}=f(r)$.

It is shown in the Appendix that at every point of an orbit, except possibly at its apses, the curvature is given by the formula in (50). Thus, for any portion of the orbit not containing an apse, (39) may be written as a first-order differential equation ${ }^{4}$

$\frac{h^{2}}{p^{3}} \frac{d p}{d r}=f(r)$.

\footnotetext{
${ }^{4}$ The key formula (40) is attributed to Abraham de Moivre, although a similar result may have been previously known to Newton. De Moivre communicated it in a letter to Johann Bernoulli in 1705; Bernoulli responded with a proof in 1706, which he later published. For more details, see the historical study [10].
}

If $f(r)$ is integrable, we obtain

$\frac{1}{2} \frac{h^{2}}{p^{2}}+\int f(r) d r=$ const.,

which is equivalent to energy conservation for the particle $P$.

Additional material on central forces may be found in [2-6].

Acknowledgements I would like to thank Professor Charles E. Smith, of Oregon State University, and Professor Nathaniel Grossman of the University of California, Los Angeles, for their comments on a draft of this note.

Open Access This article is distributed under the terms of the Creative Commons Attribution Noncommercial License which permits any noncommercial use, distribution, and reproduction in any medium, provided the original author(s) and source are credited.

\section{Appendix: The radial-perpendicular representation of planar curves}

In this appendix, some useful background material on the radial-perpendicular, or $(r, p)$, representation of planar curves is provided.

Consider a particle $P$ moving along a curve $\mathcal{C}$ in a fixed plane through $O$ (Fig. 2). $\{\mathbf{i}, \mathbf{j}\}$ is a fixed orthonormal basis and $\mathbf{k}=\mathbf{i} \times \mathbf{j}$. Choose polar coordinates and let $\left\{\mathbf{e}_{r}, \mathbf{e}_{\theta}\right\}$ be the associated basis. Clearly,

$\mathbf{x}=\mathbf{r}=r \mathbf{e}_{r}, \quad \frac{d \mathbf{e}_{r}}{d \theta}=\mathbf{e}_{\theta}, \quad \frac{d \mathbf{e}_{\theta}}{d \theta}=-\mathbf{e}_{r}$.

Suppose that $\mathcal{C}$ is specified in polar form $r=r(\theta)$. The unit tangent vector to $\mathcal{C}$ is ${ }^{5}$

$\mathbf{e}_{t}=\frac{d \mathbf{r}}{d s}=\left(\frac{d r}{d \theta} \mathbf{e}_{r}+r \mathbf{e}_{\theta}\right) \frac{d \theta}{d s}$.

Hence,

$\left[r^{2}+\left(\frac{d r}{d \theta}\right)^{2}\right]\left(\frac{d \theta}{d s}\right)^{2}=1$.

Observing that the triangle $O Z P$ in Fig. 2 is rightangled, and $\angle O P Z=\beta$, we note that $\mathbf{e}_{t}$ can also be expressed as

$\mathbf{e}_{t}=\cos \beta \mathbf{e}_{r}+\sin \beta \mathbf{e}_{\theta}=\frac{1}{r}\left(q \mathbf{e}_{r}+p \mathbf{e}_{\theta}\right)$.

\footnotetext{
${ }^{5}$ In the plane (Fig. 2), $\mathbf{e}_{t}$ may also be expressed as $\mathbf{e}_{t}=\cos \phi \mathbf{i}+$ $\sin \phi \mathbf{j}$ and $\kappa=d \phi / d s$.
} 


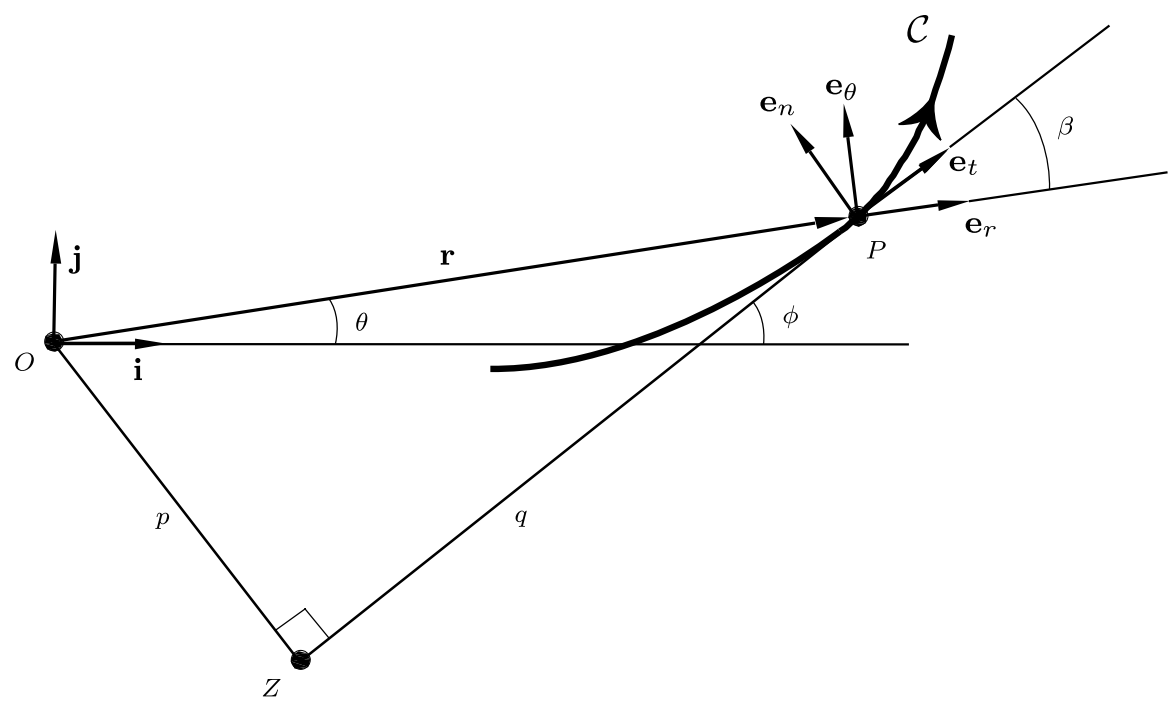

Fig. 2 Motion of a particle $P$ along a curve $\mathcal{C}$ in a fixed plane

It follows from (43) and (45) that

$p=r^{2} \frac{d \theta}{d s}, \quad q=r \frac{d r}{d \theta} \frac{d \theta}{d s}$.

By virtue of (46) 1 and (44),

$\frac{1}{p^{2}}=\frac{1}{r^{2}}+\left(\frac{1}{r^{2}} \frac{d r}{d \theta}\right)^{2}$.

The relation $(28)_{2}$ furnishes

$\frac{d p}{d s}=\kappa r \frac{d r}{d \theta} \frac{d \theta}{d s}$.

If an $(r, p)$ representation of a curve is given, (48) becomes

$\frac{d p}{d r} \frac{d r}{d \theta}=\kappa r \frac{d r}{d \theta}$.

For all points at which $d r / d \theta$ does not vanish, (49) produces a first-order differential equation for curvature:

$\kappa=\frac{1}{r} \frac{d p}{d r} \quad\left(\frac{d r}{d \theta} \neq 0\right)$.

At an apse of an orbit, the radius vector is perpendicular to the tangent, and hence $p=r, q=0, \beta=\pi / 2$; further, in view of (46) $2,(47)$ and (48), $d r / d s=0$, $d r / d \theta=0$, and $d p / d s=0$ at an apse. The limits of both sides of (50) must be examined, as the apse is approached, to check whether the equality in (50) still holds at the apse.

\section{References}

1. Siacci F (1879) Moto per una linea piana. Atti R Accad Sci Torino 14:750-760

2. Whittaker ET (1937) A treatise on the analytical dynamics of particles and rigid bodies, 4 th edn. Cambridge University Press, Cambridge. Dover, New York (1944)

3. Grossman N (1996) The sheer joy of celestial mechanics. Birkhäuser, Basel

4. Love AEH (1921) Theoretical mechanics, 3rd edn. Cambridge University Press, Cambridge

5. Ramsey AS (1933) Dynamics. Cambridge University Press, Cambridge

6. Lamb H (1923) Dynamics, 2nd edn. Cambridge University Press, Cambridge

7. http://www.torinoscienza.it/accademia/personaggi, accessed 20 October 2008

8. Siacci F (1879) Moto per una linea gobba. Atti R Accad Sci Torino 14:946-951

9. Casey J (2007) Areal velocity and angular momentum for non-planar problems in particle mechanics. Am J Phys 75:677-685

10. Guicciardini N (1995) Johann Bernoulli, John Keill and the inverse problem of central forces. Ann Sci 52:537-575 